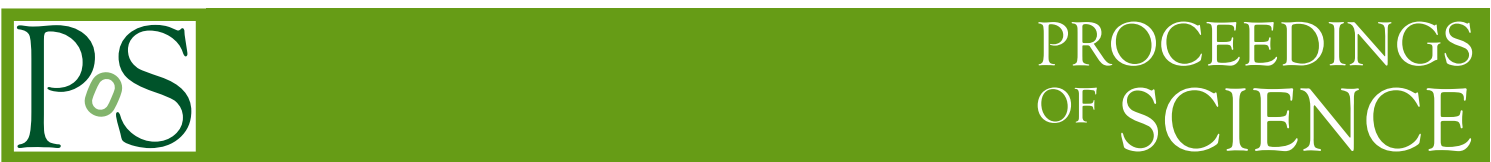

\title{
AdS/QCD at the correlator level
}

\author{
Hilmar Forkel*† \\ Institut für Physik, Humboldt-Universität zu Berlin, D-12489 Berlin, Germany \\ E-mail: forkel@ift.unesp.br
}

We derive and analyze predictions of both the hard-wall and dilaton soft-wall AdS/QCD approximations for the scalar glueball correlator and decay constants. We confront the results with QCD information from the lattice, the operator product expansion (OPE), a hypothetical UV gluon mass associated with the short-distance behavior of the heavy-quark potential, and a low-energy theorem based on the anomalous dilatational Ward identity. Both duals turn out to encode complementary aspects of the above, nonperturbative QCD physics. The OPE Wilson coefficients, in particular, are shown to provide a challenging testing ground for the impact of the strongly coupled holographic UV dynamics on dual gravity predictions.

8th Conference Quark Confinement and the Hadron Spectrum September 1-6 2008

Mainz, Germany

${ }^{*}$ Speaker.

${ }^{\dagger}$ Supported by the Deutsche Forschungsgemeinschaft. 
The first generation of holographic dual candidates for QCD [1] has reached a stage of development in which it becomes increasingly important to systematically map out their limitations, and thereby to provide a quantitative basis for identifying the most promising improvement strategies. One way of proceeding in this bottom-up fashion is to survey more detailed holographic predictions than the hadron mass spectra. As a step in this direction, we have recently derived and analyzed the predicitions of two popular AdS/QCD duals, i.e. the hard-wall [2] and dilaton soft-wall [3] backgrounds, for the $0^{++}$glueball correlation function and decay constants [ [

Both holographic duals turn out to complement each other in their representation of specific nonperturbative glueball physics (at momenta larger than the QCD scale): the soft-wall correlator

$$
\begin{aligned}
\hat{\Pi}^{(\mathrm{sw})}\left(Q^{2}\right)= & -\frac{2 R^{3}}{\kappa^{2}} \lambda^{4}\left[1+\frac{Q^{2}}{4 \lambda^{2}}\left(1+\frac{Q^{2}}{4 \lambda^{2}}\right) \psi\left(\frac{Q^{2}}{4 \lambda^{2}}\right)\right] \\
& \stackrel{Q^{2} \gg \lambda^{2}}{\longrightarrow}-\frac{2}{\pi^{2}} Q^{4}\left[\ln \frac{Q^{2}}{\mu^{2}}+\frac{4 \lambda^{2}}{Q^{2}} \ln \frac{Q^{2}}{\mu^{2}}+\frac{2^{2} 5}{3} \frac{\lambda^{4}}{Q^{4}}-\frac{2^{4}}{3} \frac{\lambda^{6}}{Q^{6}}+\frac{2^{5}}{15} \frac{\lambda^{8}}{Q^{8}}+\ldots\right]
\end{aligned}
$$

(where $\psi(z)=\Gamma^{\prime}(z) / \Gamma(z), \lambda$ is the dilaton mass scale and $R^{3} / \kappa^{2}=2\left(N_{c}^{2}-1\right) / \pi^{2}$ ) contains all known types of QCD power corrections, generated both by vacuum condensates and by a hypothetical UV gluon mass suggested to encode the short-distance behavior of the static quark-antiquark potential [5], while sizeable exponential corrections as induced by small-scale QCD instantons [6] are reproduced in the hard-wall correlator

$$
\begin{aligned}
\hat{\Pi}^{(\mathrm{hw})}\left(Q^{2}\right)= & \frac{R^{3}}{8 \kappa^{2}} Q^{4}\left[2 \frac{K_{1}\left(Q z_{m}\right)}{I_{1}\left(Q z_{m}\right)}-\ln \frac{Q^{2}}{\mu^{2}}\right] \\
& \stackrel{Q^{2} \gg z_{m}^{-2}}{\longrightarrow}-\frac{2}{\pi^{2}} Q^{4} \ln \frac{Q^{2}}{\mu^{2}}+\frac{4}{\pi}\left[1+\frac{3}{4} \frac{1}{Q z_{m}}+O\left(\frac{1}{\left(Q z_{m}\right)^{2}}\right)\right] Q^{4} e^{-2 Q z_{m}}
\end{aligned}
$$

(where the IR brane is located at $z_{m}$ ). This complementarity generalizes to other hadron channels, allows to relate holographic predictions to specific aspects of the gauge dynamics and suggests to combine the underlying brane- and dilaton-induced IR physics into improved QCD duals.

While the various contributions to the holographic estimates (1) and (12) have the expected order of magnitude, the signs of the two leading power corrections in Eq. (1) are opposite to QCD predictions and violate the factorization approximation to the four-gluon condensate. We have argued that this provides specific evidence for the short-distance physics in the Wilson coefficients to be inadequately reproduced (beyond the leading conformal logarithm) by the strongly-coupled UV dynamics of the gravity duals [ $₫$. We have further shown that this problem cannot be mended by admixing the UV-subleading solution to the bulk-to-boundary propagator (as recently advocated in Ref. [团) without loosing consistency and predictive power [8]. It remains to be seen whether $\alpha^{\prime}$ corrections, in particular the resummed, local ones which are suggested to reproduce the RG flow of the strong coupling [9], can generate improved holographic predictions for the power corrections.

Since the Wilson coefficients of the $0^{++}$glueball correlator furthermore receive unusually small perturbative QCD contributions while those from small instantons are enhanced, the hardwall correlator may produce the better overall AdS/QCD prediction. Our holographic estimates for the glueball decay constants, which probe aspects of the dual dynamics to which the mass spectrum is less sensitive and which are important for experimental glueball searches, provide 
indirect evidence for this expectation. The large hard-wall prediction $f_{S}^{(\mathrm{hw})} \simeq 0.8-0.9 \mathrm{GeV}$ for the ground-state decay constant reflects the strong instanton-induced short-distance attraction in the scalar QCD glueball correlator, implies an exceptionally small $0^{++}$glueball size and is indeed close to IOPE sum-rule [6] and lattice [10] results. On the other hand, the absence of instanton contributions in the soft wall, which was designed to reproduce confinement-induced linear Regge trajectories, agrees with QCD evidence against a direct involvement of instantons in the underlying flux-tube formation.

To summarize, our findings demonstrate that the comparison of holographic predictions with QCD information at the correlator level provides detailed insights into quantitative aspects of the gauge dynamics which different gravity duals encode. We have emphasized that information from the operator product expansion, which factorizes contributions of short- and long-distance physics, allows for a transparent analysis of typical shortcomings of holographic models which are rooted in their strongly coupled UV sector. These limitations notwithstanding, the amount of glueball dynamics which even the simplest holographic duals encode is encouraging and indicates that AdS/QCD may indeed develop into a systematically improvable approximation to QCD.

We acknowledge financial support from the Fundação de Amparo a Pesquisa do Estado de São Paulo (FAPESP) and the Deutsche Forschungsgemeinschaft (DFG).

\section{References}

[1] K. Peeters and M. Zamaklar, The string/gauge theory correspondence in QCD, Eur. Phys. J. Special Topics 152 (2007) 113 [arXiv: 0708 . 1502]; S.J. Brodsky and G.F. de Téramond, AdS/CFT and Light-Front QCD, arXiv: 0802.0514 ; J. Erdmenger, N. Evans, I. Kirsch and E. Threlfall, Mesons in gauge/gravity duals - a review, Eur. Phys. J. A 35 (2008) 81 [arXiv: 0711 . 4 467].

[2] J. Polchinski and M. J. Strassler, Hard scattering and gauge/string duality, Phys. Rev. Lett. 88 (2002) 031601 [hep-th/0109174].

[3] A. Karch, E. Katz, D.T. Son and M.A. Stephanov, Linear confinement and AdS/QCD, Phys. Rev. D 74 (2006) 015005 [hep-ph/0602229].

[4] H. Forkel, Holographic glueball structure, Phys. Rev. D 78 (2008) 025001 [arXiv : 0711.1179 ].

[5] K.G. Chetyrkin, S. Narison and V.I. Zakharov, Short-distance tachyonic gluon mass and $1 / Q^{2}$ corrections, Nucl. Phys. B 550 (1999) 353 [hep-ph/9811275].

[6] H. Forkel, Scalar gluonium and instantons, Phys. Rev. D 64 (2001) 034015 [hep-ph / 0005004 ]; Direct instantons, topological charge screening and QCD glueball sum rules, Phys. Rev. D 71 (2005) 054008 [hep-ph/0312049]; QCD glueball sum rules and vacuum topology, Proceedings of Continuous advances in QCD, Minneapolis (2006), 383 [hep-ph/0608071].

[7] P. Colangelo, F. De Fazio, F. Jugeau and S. Nicotri, Investigating AdS/QCD duality through scalar glueball correlators, arXiv:0711.4747.

[8] H. Forkel, Glueball correlators as holograms, to be published in the Proceedings of Continuous advances in QCD, Minneapolis (2008), arXiv:0808.0304.

[9] U. Gürsoy and E. Kiritsis, Exploring improved holographic theories for QCD: part I, JHEP 02 (2008) 032 [arXiv:0707.1324].

[10] Y. Chen et al., Glueball spectrum and matrix elements on anisotropic lattices, Phys. Rev. D 73 (2006) 014516 [hep-lat/0510074]. 